UCRL-7316

Nuclear Explosions-

Peaceful Applications, UC- 35

TID-4500 (19th Ed.)

UNIVERSITY OF CALIFORNIA

Lawrence Radiation Laboratory

Livermore, California

Contract No. W-7405-eng-48

THE TENSOR CODE

G. Maenchen

S. Sack

April 9, 1963

LEGAL NOTICE

This report was prepared as sn account of Government sponsored work Netther the United seates, nor the Commisesion, nor any person actung on bebnlf of the Commiselon

aey,

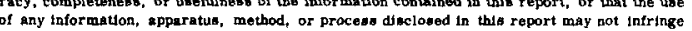

privately owned rights, or
B Assumes any liabintites with respect to the use of, or for damages resulung from the

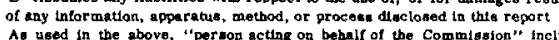

As used in the above, "perton acting on besalf of the Commission" includea any etm

such employee or contractor of the Comminston, or omployee of aych contractor prent that dissominates, or provides access to, any tnformation purenent to hlo employment or contract with the Commiasion, or bis employment with euch contrector 
Printed in USA. Price 75 cents. Available from the office of Technical Services U. S. Department of Commerce Washington 25, D.C. 


\section{DISCLAIMER}

This report was prepared as an account of work sponsored by an agency of the United States Government. Neither the United States Government nor any agency Thereof, nor any of their employees, makes any warranty, express or implied, or assumes any legal liability or responsibility for the accuracy, completeness, or usefulness of any information, apparatus, product, or process disclosed, or represents that its use would not infringe privately owned rights. Reference herein to any specific commercial product, process, or service by trade name, trademark, manufacturer, or otherwise does not necessarily constitute or imply its endorsement, recommendation, or favoring by the United States Government or any agency thereof. The views and opinions of authors expressed herein do not necessarily state or reflect those of the United States Government or any agency thereof. 


\section{DISCLAIMER}

Portions of this document may be illegible in electronic image products. Images are produced from the best available original document. 


\title{
THE TENSOR CODE
}

G. Maenchen and S. Sack

Lawrence Radiation Laboratory, University of California Livermore, California

April 9, 1963

\begin{abstract}
A numerical technique for the calculation of underground explosion phenomena and other time dependent problems involving the motion of elastic, plastic, fractured, and fluid materials is described. The method allows treatment of transient phenomena in two space dimensions (cartesian or axial symmetry), and is particularly appropriate for problems involving compres sible flow, large displacements, and free transitions of material between the elastic, cracked, plastic, and fluid states. The equations of motion are a straightforward generalization (to irclude a stress tensor) of conventional Lagrangian techniques for compressible flow and shock hydrodynamics problems. The methods of defining the stress system which have been used or are readily available can include the following:

1. General nonlinear viscoelasticity with treatment of fractures.

2. Plastic flow as defined by a variety of yield conditions including Tresca, von Mises and Coulomb yield surfaces, work hardening, temperature dependence and strain-rate effects.

3. Compressible fluid flow with or without shocks.

4. Free transitions between all material states.
\end{abstract}

They are limited only by the user's imagination and experimental knowledge of the properties of real materials. 


\title{
THE TENSOR CODE
}

\author{
G. Maenchen and S. Sack \\ Lawrence Radiation Laboratory, University of California \\ Livermore, California \\ April 9, 1963
}

\section{INTRODUCTION}

To predict underground explosion phenomena without merely scaling past explosions, a method of calculating the motion of elastic, plastic, and fluid materials is required. Such a calculation involves the solution of a set of coupled partial differential equations derived from the conservation laws for mass, momentum, and energy together with a reasonable mathematical model of the response of the materials concerned. ${ }^{1}$ Analytic solutions for nontrivial cases are difficult and frequently impossible to obtain. At the Lawrence Radiation Laboratory (Livermore), two "codes" have been developed which integrate these equations numerically on digital computers. The calculations have been simplified by requiring a degree of symmetry in the problem. The older code, 2 UNEC, describes spherically symmetric motion; i.e., it is one-dimensional in that there is only one (radial) permitted direction of motion. This report will describe a two-dimensional code, TENSOR, which was developed to study cylindrically symmetric problems (e. g. , craters) where two spatial variables are required.

The TENSOR equations describe the motion of material under the influence of a stress tensor (in contrast to the usual scalar pressure in hydrodynamic calculations). In TENSOR, the differential equations of motion are replaced by a set of finite difference equations: the continuous spatial

In the immediate vicinity of a violent explosion the problem may be simplified by ignoring elastic and plastic effects. Calculations of explosions in such a fluid medium have been described by H. L. Brode and R. L. Bjork in RAND Report RM-2600 (1960).

${ }^{2}$ J. H. Nuckolls, Lawrence Radiation Laboratory (Livermore) Report UCRL-5675, Part I, pp. 120-134. 
distribution of stress, velocity, etc., are approximated by a set of values defined at discrete positions (zones) in the material. Since a Lagrangian form is used, these zones describe specific pieces of the moving material and move with it. An example of such a mesh of zones is shown in Fig. 1 . The continuous time variation of the stress, velocity, etc., is similarly approximated by values defined at discrete intervals in time.

The calculation integrates the equation of motion in a series of small time steps. At some time $t$, the stress field is known from the distribution of values of stress in each zone and the positions of these zones. The momentum conservation equation is used to calculate the acceleration of each mesh point. This acceleration, acting for a small time step $\Delta t$, alters the previous velocities, and the new velocity and position of each mesh point is found at $t+\Delta t$. A generalized Hooke's law or some other equation of state is used to calculate the stress at $t+\Delta t$. At this point, the distinction between elastic, fractured, plastic, and fluid materials is made by imposing certain restrictions on the stress tensor. Having calculated the new stress in each zone, we may now repeat the entire process. A typical cratering problem involves a mesh of approximately 1000 zones and approximately 1000 time steps, and requires about 4 hours on the IBM 7090. At this point, the surface motion is such that zone distortion becomes serious enough to reduce the time step $\Delta t$ to an unprofitable level.

No attempt has been made to derive the equations in a rigorous fashion. In some instances (e.g., the form and use of the artificial viscosity), the choice was based on a mixture of intuition and experience with other codes and was then justified empirically. However, the Lagrangian formulation of the momentum and energy equations (in difference form) together with such features as the weighting factors has been used successfully on many other codes and forms a very convenient and flexible framework. Within this framework many possible "constitutive" equations may be used. In TENSOR these comprise the stress calculation together with the various adjustments for plastic flow and crack formation. The general framework would permit the inclusion of many other effects such as heat conduction, shock-induced chemical reactions including detonation waves, and so on.

Finally, two basic limitations of the code must be mentioned. Stability considerations require that the time step $\Delta t$ used in the calculation be less 


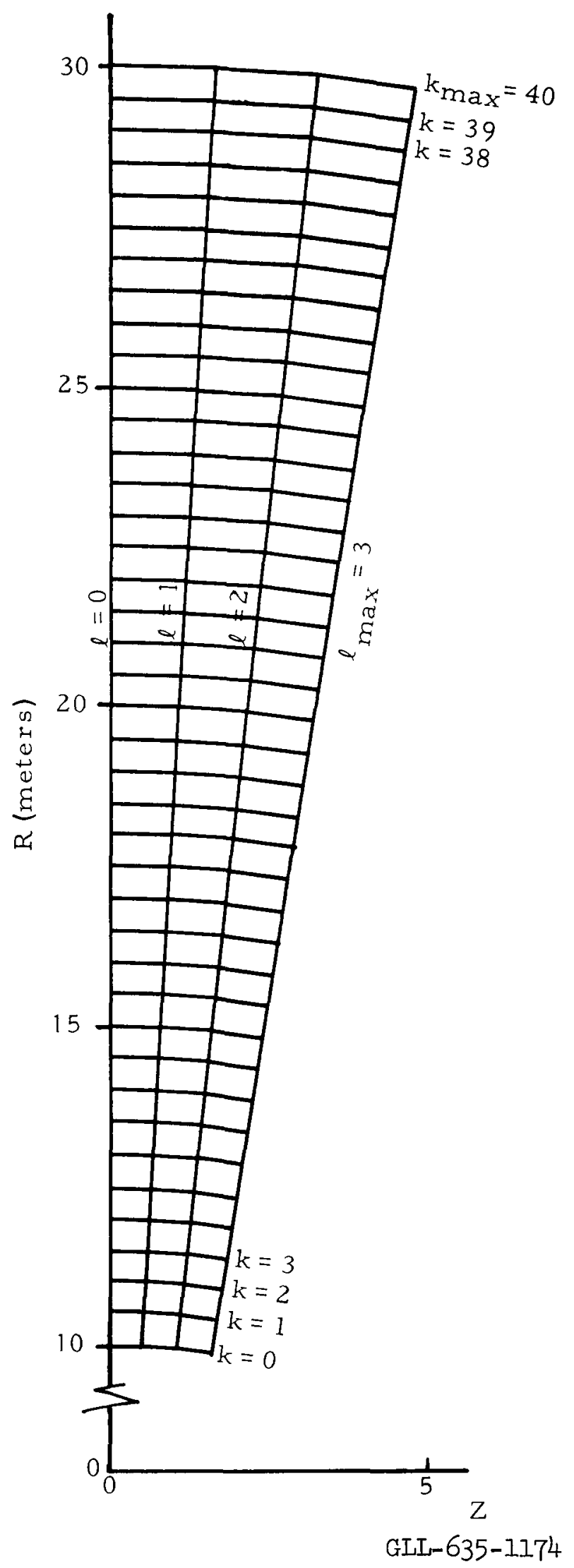

Fig. 1. TENSOR problem A. 
than the ratio (zone size)/(sound speed). Practical considerations thus limit the code to transient phenomena where the time scale is limited to a few sound reverberation times across the material being considered. A second limitation is that the Lagrangian mesh, while flexible, can not be distorted without limit. Thus the mesh can not follow material in turbulent flow (although moderate distortions can be handled by "rezoning" procedures).

\section{DEFINITIONS AND NOTATION}

The familiar forms of equation of motion, definitions of strain rate, etc., are normally expressed in terms of Eulerian coordinates. The difference equations used in TENSOR are written in terms of Lagrangian coordinates. The Eulerian derivatives, therefore, must be expressed in Lagrangian form.

Lagrangian coordinates: $k, \ell$

Eulerian coordinates: $\quad R(k, \ell, t)=(\text { radial position })^{*}$ of the mesh point given by $k, l$ at time $t$

$Z(k, \ell, t)=$ axial position.

For any function $F$, we may write:

$$
\begin{aligned}
& \frac{\partial F}{\partial k}=\frac{\partial F}{\partial R} \frac{\partial R}{\partial k}+\frac{\partial F}{\partial Z} \frac{\partial Z}{\partial k} \\
& \frac{\partial F}{\partial l}=\frac{\partial F}{\partial R} \frac{\partial R}{\partial \ell}+\frac{\partial F}{\partial Z} \frac{\partial Z}{\partial l}
\end{aligned}
$$

from which we obtain the Lagrangian expression for the Eulerian derivatives:

$$
\begin{aligned}
& \frac{\partial F}{\partial \mathrm{R}}=\frac{1}{\mathrm{~J}}\left(\frac{\partial F}{\partial \mathrm{k}} \frac{\partial Z}{\partial \ell}-\frac{\partial F}{\partial \ell} \frac{\partial \mathrm{Z}}{\partial \mathrm{k}}\right) \\
& \frac{\partial \mathrm{F}}{\partial \mathrm{Z}}=\frac{-1}{\mathrm{~J}}\left(\frac{\partial \mathrm{F}}{\partial \mathrm{k}} \frac{\partial \mathrm{R}}{\partial \ell}-\frac{\partial F}{\partial \ell} \frac{\partial \mathrm{R}}{\partial \mathrm{k}}\right)
\end{aligned}
$$

where

$$
\mathrm{J}=\frac{\partial \mathrm{R}}{\partial \mathrm{K}} \frac{\partial \mathrm{Z}}{\partial \ell}-\frac{\partial \mathrm{R}}{\partial \ell} \frac{\partial \mathrm{Z}}{\partial \mathrm{k}}=\text { Jacobian of the transformation. }
$$

In the code, the Lagrangian variables $k$ and $\ell$ take on discrete values $(0,1,2, \ldots)$. They will be used as subscripts to indicate the mesh point

\footnotetext{
* The code has provision for two-dimensional cartesian calculation in which case $R$ becomes a cartesian coordinate.
} 
at which some variable (e. $g ., R$ ) is defined. A discrete time variable, $n$, will be used as a superscript:

$$
t^{n}=t^{n-1}+\Delta t^{n-1 / 2} \text {. }
$$

Thus,

$$
R(k, \ell, t)=R_{k, \ell}^{n}=\underset{\text { at time } t^{n} .}{\text { atial position of mesh point given by } k \text { and } \ell}
$$

In order to make the difference equations less cumbersome, the following abbreviated notation will be used whenever possible:

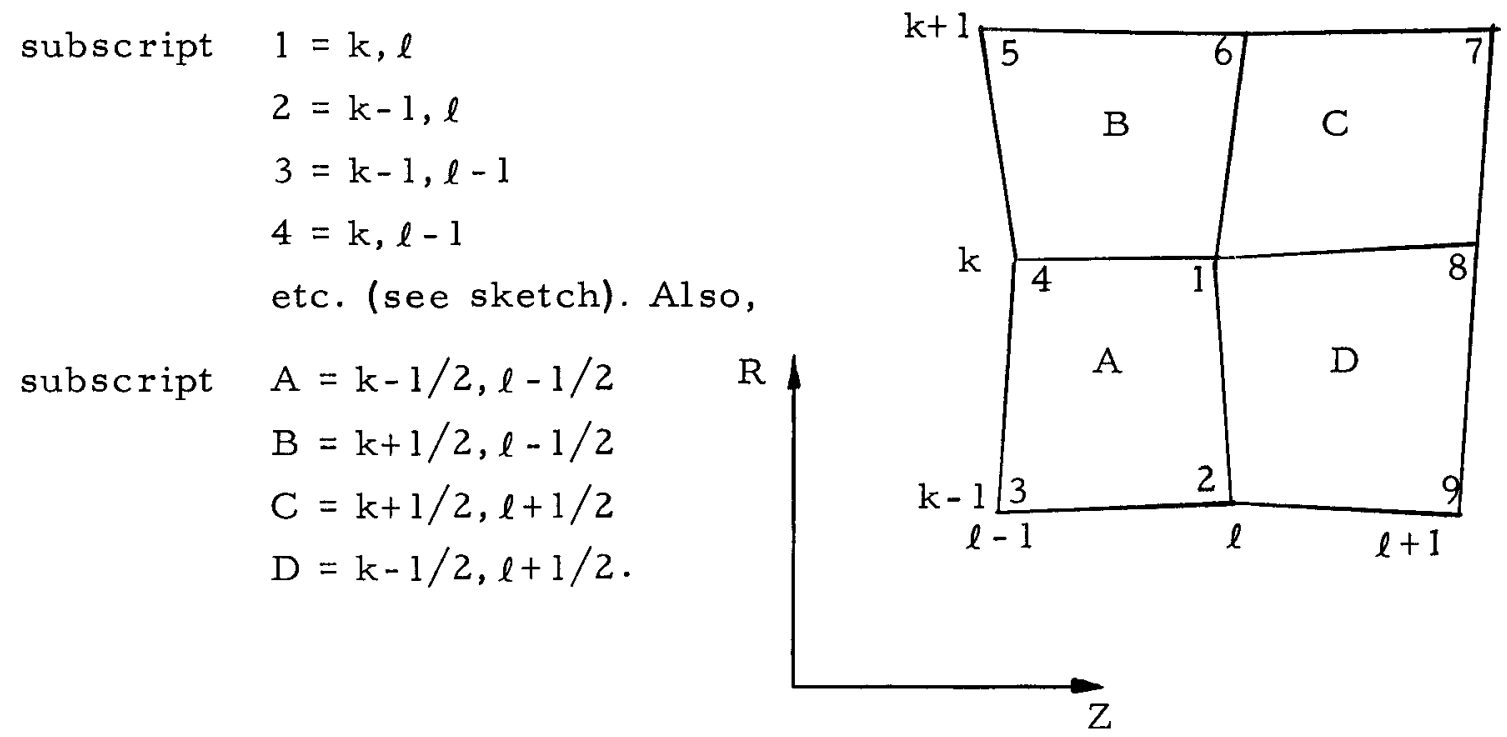

The following variables are defined at mesh points:

$$
\begin{aligned}
R_{1}^{n} & =R_{k, l}^{n}=\text { radial coordinate } \\
Z_{1}^{n} & =\text { axial coordinate } \\
u_{1}^{n-1 / 2} & =\dot{R}_{1}=\text { radial velocity component } \\
v_{1}^{n-l / 2} & =\dot{Z}_{1}=\text { axial velocity component }
\end{aligned}
$$




$$
\begin{aligned}
& \dot{\mathrm{u}}_{1}^{\mathrm{n}}, \dot{\mathrm{v}}_{\mathrm{l}}^{\mathrm{n}}=\text { radial and axial acceleration components } \\
& \Delta \mathrm{R}_{1}^{\mathrm{n}}, \Delta \mathrm{Z}_{1}^{\mathrm{n}}=\text { displacement components. }
\end{aligned}
$$

The remaining variables are defined in the mesh zones,

$$
\begin{aligned}
& \mathrm{J}_{\mathrm{A}}^{\mathrm{n}}=\mathrm{J}_{\mathrm{k}-1 / 2, \ell-1 / 2}^{\mathrm{n}}=\mathrm{Jacobian}=\text { zone area } \\
& \mathrm{V}_{\mathrm{A}}^{\mathrm{n}}=\text { zone volume (per radian) } \\
& V_{A}^{0}=\text { initial }(t=0) \text { zone volume } \\
& \delta V_{A}^{n}=V_{A}^{n}-V_{A}^{0} \\
& \mathrm{M}_{\mathrm{A}}=\text { zone mass } \\
& \rho_{A}^{n}=\text { density } \\
& \mathrm{E}_{\mathrm{A}}^{\mathrm{n}}=\text { internal energy/unit original volume } \\
& \mathrm{P}_{\mathrm{A}}^{\mathrm{n}}=\text { pressure } \\
& \left(\tau_{i}\right)_{A}^{n}=\text { components of deviatoric stress tensor }(i=R, Z, R Z) \\
& \mathrm{Q}_{\mathrm{A}}^{\mathrm{n}-1 / 2}=\text { scalar form of artificial viscosity } \\
& \left(Q_{i}\right)_{A}^{n-1 / 2}=\text { components of deviatoric artificial viscosity }(i=R, Z, R Z) \\
& \left(Q_{\ell}\right)_{A}^{n-1 / 2},\left(Q_{k}\right)_{A}^{n-1 / 2}=\text { additional damping terms } \\
& I_{A}=\text { label for material in zone } \mathrm{A} \\
& \phi_{\mathrm{A}}^{\mathrm{n}}=\text { fracture orientation angle } \\
& \left(E_{i}\right)_{A}^{n}=\text { fractional crack width }(i=1,2,3) \text {. }
\end{aligned}
$$

The time superscript will frequently be omitted. 


\section{MOMENTUM EQUATION}

In a system with cylindrical symmetry (i.e., one where all derivatives with respect to the azimuthal coordinate $\theta$ vanish) $)^{*}$, the stress tensor can have only four independent nonzero elements:

$$
(\tau)=\left(\begin{array}{lll}
{ }^{\tau} \mathrm{RR} & 0 & { }^{\tau} \mathrm{RZ} \\
0 & { }^{\tau} \theta \theta & 0 \\
{ }^{\tau}{ }_{\mathrm{RZ}} & 0 & { }^{\tau} \mathrm{ZZ}
\end{array}\right)
$$

Here $\tau_{R R}$ is the (tensile) force acting in the $R$ direction on a unit element of area oriented normal to the $R$ direction. ${ }^{3}$ The shear stress, $\tau_{R Z}$, is the force in the $R$ direction acting on an element normal to the $Z$ axis. (By symmetry, $\tau_{\mathrm{RZ}}=\tau_{\mathrm{ZR}} \cdot$ )

To facilitate the calculation of plastically deformed material, the stress is expressed as the sum of an isotropic and deviatoric part, 4 because plastic yielding limits only the effective shear stress but not the average pressure.

$$
(\tau)=\left(\begin{array}{rrr}
-\mathrm{P} & 0 & 0 \\
0 & -\mathrm{P} & 0 \\
0 & 0 & -\mathrm{P}
\end{array}\right)+\left(\begin{array}{lll}
\tau_{\mathrm{R}} & 0 & \tau_{\mathrm{RZ}} \\
0 & \tau_{\theta} & 0 \\
\tau_{\mathrm{RZ}} & 0 & \tau_{\mathrm{Z}}
\end{array}\right)
$$

where

$$
\begin{aligned}
P & =-\frac{1}{3}\left(\tau_{R R}+\tau_{\theta \theta}+\tau_{Z Z}\right) \\
\tau_{R} & =\tau_{R R}+P \\
\tau_{Z} & =\tau_{Z Z}+P \\
\tau_{\theta} & =\tau_{\theta \theta}+P=-\left(\tau_{R}+\tau_{Z}\right)
\end{aligned}
$$

This notation, though customary in plastic flow calculations, is inconsistent in sign. $P$ is positive for compressed material while $\tau_{i i}$ and $\tau_{i}$ are positive for tension.

\footnotetext{
* In the case of cartesian calculation, $\theta$ is the third (symmetric) coordinate.

${ }^{3}$ I. S. Sokolnikoff, Mathematical Theory of Elasticity (McGraw-Hill Book Company, New York, 1946).

4 R. Hill, Mathematical Theory of Plasticity (Oxford University-Clarendon Press, London, 1950).
} 
In terms of these variables, the momentum equation is,

$$
\begin{aligned}
& \dot{\mathrm{u}}=-\frac{1}{\rho} \frac{\partial\left(\mathrm{P}-\tau_{\mathrm{R}}\right)}{\partial \mathrm{R}}+\frac{1}{\rho} \frac{\partial \tau_{\mathrm{R} Z}}{\partial \mathrm{Z}}+\frac{2 \tau_{\mathrm{R}}+\tau_{\mathrm{Z}}}{\rho \mathrm{R}}+\mathrm{G}_{\mathrm{R}} \\
& \dot{\mathrm{v}}=-\frac{1}{\rho} \frac{\partial\left(\mathrm{P}-\tau_{\mathrm{Z}}\right)}{\partial \mathrm{Z}}+\frac{1}{\rho} \frac{\partial \tau_{\mathrm{R} Z}}{\partial \mathrm{R}}+\frac{{ }^{\tau} \mathrm{RZ}}{\rho \mathrm{R}}+\mathrm{G}_{\mathrm{Z}}
\end{aligned}
$$

The final term in each expression represents a body force and is used to include gravity effects (normally the mesh is oriented so $G_{Z}=g$ or $-g$, $\mathrm{G}_{\mathrm{R}}=0$ ). To prevent oscillation, it was necessary to introduce some damping analogous to the artificial viscosity first proposed for numerical calculations of shock hydrodyamics by Von Neumann and Richtmyer. 5 In the present case, this viscosity is generalized to a tensor and separated into an isotropic and a deviatoric part. Thus, $P$ is replaced by $P+Q, \tau_{R}$ by $\tau_{R}+Q_{R}$, etc.

The acceleration at the Lagrangian mesh point $k, \ell$ must now be expressed in terms of the stress in the surrounding four zones. The modifications required at the edges of the Lagrange mesh will be discussed separately. We note that the acceleration equations contain both derivative terms (which are really defined at zone boundaries) and nonderivative terms which are defined in the zones.

Consider first the nonderivative terms. * We may define an average radius in a zone as $\bar{R}_{A}^{n}=V_{A}^{n} / J_{A}^{n}$ so $1 / \rho R=V J / M V=J / M$. Thus, the contribution of the nonderivative forms to the acceleration in each zone is

$$
\begin{aligned}
& (\dot{u})_{i}=\frac{J_{i}}{M_{i}}\left(2 \tau_{R}+2 Q_{R}+\tau_{Z}+Q_{Z}\right)_{i} \\
& (\dot{v})_{i}=\frac{J_{i}}{M_{i}}\left(\tau_{R Z}+Q_{R Z}\right)_{i} \quad i=A, B, C, D
\end{aligned}
$$

The derivative terms are evaluated separately at each of the boundaries between the adjacent zones. Consider the boundary between zones C and D. Here we can have only $k$ derivatives of stress so the radial acceleration due

\footnotetext{
5 J. Von Neumann and R. D. Richtmyer, J. Appl. Phys. 21, 232 (1950).

* These terms correspond to acceleration due to hoop stress. They are zero in the cartesian case.
} 
to gradients across this interface $(k, \ell$ to $k, \ell+1)$ is $\left.u_{81}=\frac{-1}{(\rho J)_{81}} \frac{\partial}{\partial k}: P+Q-\tau_{R}-Q_{R} \cdot \frac{\partial Z}{\partial \ell}+\frac{\partial}{\partial k}:{ }_{R Z}+Q_{R Z} \frac{\partial R}{\partial l}\right)$ at 81 boundary. At this point one must choose among various possible methods of differencing. Since the stresses are defined within the zones, we use the simplest choice:

$\left[\frac{\partial}{\partial \mathrm{k}}\left(\right.\right.$ stress ${ }^{1} \mathrm{k}, \ell+1 / 2=(\text { stress })_{k+1 / 2, \ell+1 / 2}-(\operatorname{stress})_{k-1 / 2, \ell+1 / 2}$

which we abbreviate to (stress) C-D. The simplest choice for the coordinate difference, $(\partial Z / \partial \ell)_{k_{s} \ell+1 / 2}=Z_{k, \ell+1}-Z_{k, \ell}$, was used at first. While this was satisfactory for a mesh of rectangular zones, it led to errors in a mesh having trapezoidal zones, because $1 / \mathrm{J})(\partial \mathrm{Z} / \partial \ell)$ is essentially equivalent to $1 / \triangle \mathrm{R}$, the effective distance between zone centers.

Thus, this simple coordinate difference led to arbitrarily small accelerations when the common boundary between zones became vanishingly small. Consequently, an average coordinate difference is now used in the code:

$k+1$
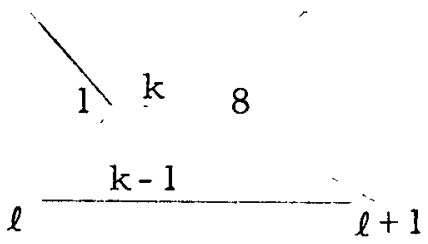

$$
\begin{aligned}
(X & =R \text { or } Z) \\
\bar{x}_{81} & =\left(\frac{\partial X}{\partial l}\right)_{k, l+1 / 2}=\frac{1}{4} \cdot 2\left(x_{8}-x_{1}\right)+x_{7}-x_{6}+x_{9}-x_{2} \\
\bar{x}_{61} & =\left(\frac{\partial x}{\partial k}\right)_{k+1 / 2, \ell}=\frac{1}{4}-2\left(x_{6}-x_{1}\right)+x_{7}-x_{8}+x_{5}-x_{4}
\end{aligned}
$$

A simple average is used for the density times area term:

$$
\begin{aligned}
& (\rho J)_{81}=\frac{1}{2}\left[\left(\frac{M}{V} J\right)_{C}+\left(\frac{M}{V} J\right)_{D}\right. \\
& \left.(\rho J)_{61}=\frac{1}{2}\left(\frac{M}{V} J\right)_{C}^{\prime}+\left(\frac{M}{V} J\right)_{B}\right]
\end{aligned}
$$

In terms of these average differences, we obtain

$$
\dot{u}_{81}=\frac{-1}{(\rho J)_{81}}\left[\left(P+Q-\tau_{R}-Q_{R}\right)_{C-D} \bar{Z}_{81}+\left(\tau_{R Z}+Q_{R Z}{ }^{\prime} C-D \bar{R}_{81}+\right.\text { q term }\right.
$$




$$
\begin{aligned}
& \dot{v}_{81}=\frac{1}{(\rho J)_{81}}\left[\left(P+Q-\tau_{Z}-Q_{Z}\right)_{C-D} \bar{R}_{81}+\left(\tau_{R Z}+Q_{R Z}\right)_{C-D} \bar{Z}_{81}\right]+q \operatorname{term} \\
& \dot{\mathrm{u}}_{61}=\frac{1}{(\rho J)_{61}}\left[\left(P+Q-\tau_{R}-Q_{R}\right)_{C-B} \bar{Z}_{61}+\left(\tau_{R Z}+Q_{R Z}\right)_{C-B} \bar{R}_{61}\right]+\text { q term } \\
& \dot{v}_{61}=\frac{-1}{(\rho J)_{61}}\left[\left(P+Q-\tau_{Z}-Q_{Z}\right)_{C-B} \bar{R}_{61}+\left({ }^{\prime}{ }_{R Z}+Q_{R Z}{ }_{C-B} \bar{Z}_{61}\right]+q \operatorname{term} .\right.
\end{aligned}
$$

The final "q term" provides additional damping and will be described later. Similar expressions could be written for the 12 and 14 sides. These, however, need not be calculated explicitly since they may be saved from the calculation at previous mesh points (i.e., $\dot{u}_{14}$ was calculated previously as $\dot{\mathrm{u}}_{81}$ when the code was at $\mathrm{k}, \ell-1$; similarly $\dot{\mathrm{u}}_{12}$ was done as $\dot{\mathrm{u}}_{61}$ at point $k-1, \ell)$.

These acceleration terms must now be combined to give the acceleration at point $\mathrm{k}, \boldsymbol{l}$. A weighted average is used which gives the greatest weight to smaller zones (because the centers are nearer the point $k, l$ ). Thus, in a case like the one in the sketch, greater weight is given

to $\dot{\mathrm{u}}_{8 \mathrm{l}}$ and less weight to $\dot{\mathrm{u}}_{14}$. We define weight factors:

$W_{81}=\frac{\left(\overline{\mathrm{R}}_{81}\right)\left(\overline{\mathrm{R}}_{81}+\overline{\mathrm{R}}_{14}\right)+\left(\overline{\mathrm{Z}}_{81}\right)\left(\overline{\mathrm{Z}}_{81}+\overline{\mathrm{Z}}_{84}\right)}{\left(\overline{\mathrm{R}}_{81}+\overline{\mathrm{R}}_{14}\right)^{2}+\left(\overline{\mathrm{Z}}_{81}+\overline{\mathrm{Z}}_{84}\right)^{2}}$

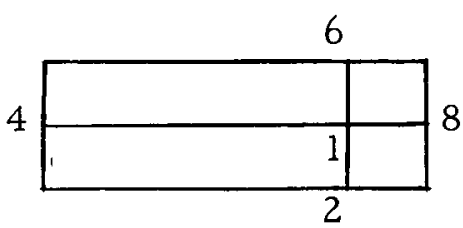

$w_{61}=\frac{\left(\overline{\mathrm{R}}_{61}\right)\left(\overline{\mathrm{R}}_{61}+\overline{\mathrm{R}}_{12}\right)+\left(\overline{\mathrm{Z}}_{61}\right)\left(\overline{\mathrm{Z}}_{61}+\overline{\mathrm{Z}}_{12}\right)}{\left(\overline{\mathrm{R}}_{61}+\overline{\mathrm{R}}_{12}\right)^{2}+\left(\overline{\mathrm{Z}}_{61}+\overline{\mathrm{Z}}_{12}\right)^{2}}$

where $\bar{R}_{61}$ etc., are the average coordinate differences defined previously. We now use $W_{81}$ as the weight of $\dot{u}_{14}$ and $\left(1-W_{81}\right)$ as the weight of $\dot{u}_{81}$. The same weighting factors will be used for the nonderivative terms so that $W_{81} W_{61}$ is the weight of zone $A,\left(1-W_{81}\right)\left(W_{61}\right)$ is the weight of zone $D$, etc. Combining all terms, we finally obtain the acceleration of point $k, \ell$ :

$$
\begin{aligned}
\dot{\mathrm{u}}_{\mathrm{k}, l}^{\mathrm{n}}=\mathrm{w}_{61} \mathrm{w}_{81}\left(\dot{\mathrm{u}}_{A}-\dot{\mathrm{u}}_{B}\right. & \left.+\dot{\mathrm{u}}_{C}-\dot{\mathrm{u}}_{\mathrm{D}}\right)+\mathrm{w}_{81}\left(\dot{\mathrm{u}}_{14}-\dot{\mathrm{u}}_{81}+\dot{\mathrm{u}}_{B}-\dot{\mathrm{u}}_{C}\right) \\
& +\mathrm{w}_{61}\left(\dot{\mathrm{u}}_{12}-\dot{\mathrm{u}}_{61}+\dot{\mathrm{u}}_{\mathrm{D}}-\dot{\mathrm{u}}_{C}\right)+\dot{\mathrm{u}}_{81}+\dot{\mathrm{u}}_{61}+\dot{\mathrm{u}}_{C}+\mathrm{G}_{\mathrm{R}}
\end{aligned}
$$


and the same form for $\dot{v}_{k, l}$. In these equations, all the quantities (stress, coordinates, volumes, etc.) were at time cycle $n$ (although the $Q$ terms were at $n-1 / 2$ ). Hence the $\dot{u}$ were at cycle $n$. Finally, the velocities and coordinates are advanced:

$$
\begin{aligned}
& u_{k, l}^{n+l / 2}=u_{k, l}^{n-1 / 2}+(\Delta t)^{n} \dot{u}^{n} ; \text { same for } v \\
& R_{k, l}^{n+1}=R_{k}^{n}+(\Delta t)^{n+1 / 2} u^{n+1 / 2} ; \text { same for } Z .
\end{aligned}
$$

\section{BOUNDARY CONDITIONS}

The equations of motion described in the previous section apply to a point in the interior of the Lagrangian mesh. At the edges of the mesh, some of these terms ( $\rho J$, $\dot{u}$, etc.) must be modified. At each of the four boundaries $\left(\mathrm{k}=0, \mathrm{k}=\mathrm{k}_{\text {max }}, \ell=0, \ell=\ell_{\text {max }}\right)$, we may have one of two possible boundary conditions. These are a rigid (smooth) wall and a free surface.

At a rigid wall, the material may slide along the surface of the wall but may not move normal to the surface. One use for the rigid wall boundary condition is at the axis of symmetry $(R=0)$ in a cylindrically symmetric problem. The effect of the smooth wall is equivalent to that of a reflected phantom zone beyond the wall where the displacement of material in the phantom zone is just the mirror image of that in the real zone. If we consider a set of axes $i, j$ parallel and normal to the wall, the "normal" stress components ${ }^{\tau}$ ii and $\tau_{j j}$ reflect symmetrically and the shear component $\tau_{i j}$ reflects antisymmetrically. We need the difference $\tau_{i j}-\tau_{i j}^{\prime}=2 \tau_{i j}$ to find the only gradient

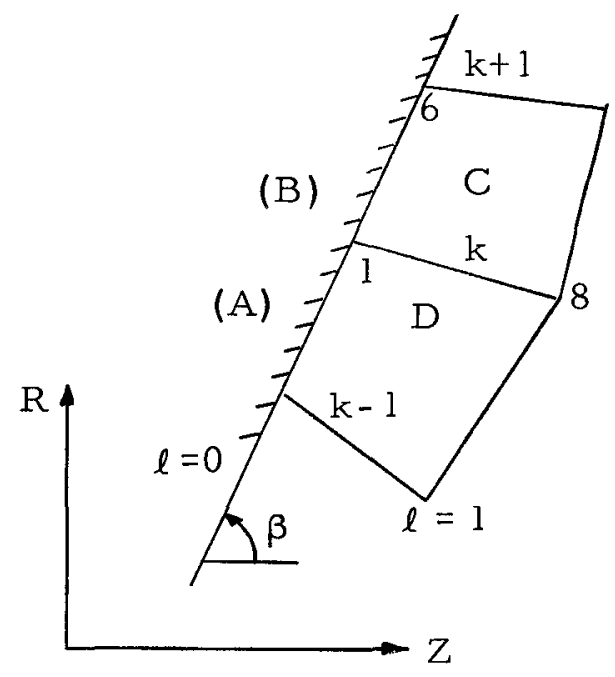
term at the wall. 


$$
\tau_{i j}=(\sin \beta \cos \beta)\left(\tau_{Z}-\tau_{R}\right)+\left(\sin ^{2} \beta-\cos ^{2} \beta\right) \tau_{R Z} .
$$

The same relation holds for the damping tensor (Q).

The portion of the acceleration at the wall due to the normal gradient of shear stress is evaluated as follows. Let

$$
F=\frac{2 V}{M J}\left[\sin \beta \cos \beta\left(\tau_{R}+Q_{R}-\tau_{Z}-Q_{Z}\right)+\left(\cos ^{2} \beta-\sin ^{2} \beta\right)\left(\tau_{R Z}+Q_{R Z}\right)\right]
$$

where all zone quantities, ( $M, V, J, \tau_{R}$, etc.) refer to the "real zone," and $\beta$ is the angle between the rigid wall and the axis. We find

at $\ell=0, \dot{\mathrm{u}}_{61}=-\overline{\mathrm{R}}_{61} \mathrm{~F}$ and

$$
\dot{v}_{61}=-\bar{Z}_{61} F \text { where the "real zone" is zone } C \text {, }
$$

at $\mathrm{k}=0, \dot{\mathrm{u}}_{81}=\overline{\mathrm{R}}_{81} \mathrm{~F}$ and

$$
\dot{\mathrm{v}}_{81}=\overline{\mathrm{Z}}_{81} \mathrm{~F} \text { where "real zone" is zone } \mathrm{C} \text {, }
$$

and similar forms at $\ell=\ell_{\max }$ and $k=k_{\max }$. These acceleration components must then be combined with internal acceleration terms and the resulting acceleration vector projected onto the rigid wall. At $\ell=0$, for example, $\dot{\mathrm{u}}_{81}, \dot{\mathrm{u}}_{\mathrm{C}}$ and $\dot{\mathrm{u}}_{\mathrm{D}}$ are calculated as for any interior mesh point while $\dot{\mathrm{u}}_{61}$ and the saved quantity $\dot{\mathrm{u}}_{12}$ are found by the rigid wall form given above. Since there is no contribution from $\dot{u}_{14}$ (which is beyond the wall), the acceleration sum is calculated with $\mathrm{W}_{81}$ set to zero. Only the component of acceleration parallel to the wall is retained. Thus,

$$
\begin{aligned}
\overrightarrow{\Delta v} & =(\dot{u} \sin \beta+\dot{v} \cos \beta) \Delta t^{n} \\
u^{n+1 / 2} & =u^{n-1 / 2}+(\overrightarrow{\Delta v}) \sin \beta \\
v^{n+1 / 2} & =v^{n-1 / 2}+(\overrightarrow{\Delta v}) \cos \beta .
\end{aligned}
$$

At a free surface, the calculation proceeds as if there were a massless phantom zone beyond the boundary. A time dependent pressure may be inserted into this phantom zone if desired. All other stress terms are zero so that the phantom zone does not contribute to the nonderivative acceleration terms (e.g., $\dot{u}_{A}$ ). The acceleration is calculated as for an interior mesh point except that one of the weight factors is modified. At $\ell=0$, for example, $\mathrm{W}_{81}$ is set to zero since there can be no acceleration due to gradients across the interface between two phantom zones. 


\section{VOLUME AND STRAIN RATE}

In the previous sections the motion of the material under the influence of the existing stress field was calculated. We must now find how this motion, in turn, alters the stress. Since we wish to permit plastic deformation, the stress-strain relation will be used in incremental form. This is because one cannot define a meaningful absolute strain (in terms of coordinates at $t=0$ ) in a material which has at some previous time behaved inelastically.

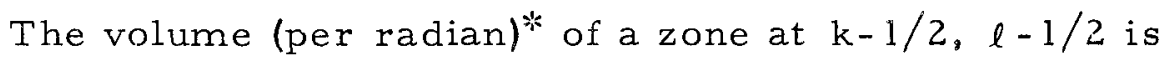

$$
\begin{aligned}
V=\frac{1}{6}\left\{\left(R_{1}+R_{2}+R_{3}\right)\right. & \left|\begin{array}{r}
\left(R_{1}-R_{2}\right)\left(R_{1}-R_{3}\right) \\
\left(Z_{1}-z_{2}\right)\left(z_{1}-z_{3}\right)
\end{array}\right| \\
& \left.+\left(R_{1}+R_{3}+R_{4}\right) \mid \begin{array}{l}
\left(R_{1}-R_{3}\right)\left(R_{1}-R_{4}\right) \\
\left(z_{1}-z_{3}\right)\left(z_{1}-z_{4}\right)
\end{array}\right\}
\end{aligned}
$$

This is calculated explicitly only at the beginning of a problem (using $R$ and $\mathrm{Z}$ at $\mathrm{t}=0$ ). Thereafter, we calculate the change in volume (and change in strain) from cycle $n$ to cycle $n+1$ in terms of time-centered coordinates at $\mathrm{n}+1 / 2$ :

$$
R=R^{n+1 / 2}=R^{n}+\frac{1}{2} u^{n+1 / 2}(\Delta t)^{n+1 / 2} ; \text { same for } Z
$$

Let $\left(x_{123}\right)=\left(x_{1}+x_{2}+x_{3}\right)$;

$$
\left|\begin{array}{c}
X \\
Y \\
123
\end{array}\right|=\left|\begin{array}{l}
\left(X_{1}-X_{2}\right)\left(X_{1}-X_{3}\right) \\
\left(Y_{1}-Y_{2}\right)\left(Y_{1}-Y_{3}\right)
\end{array}\right|
$$

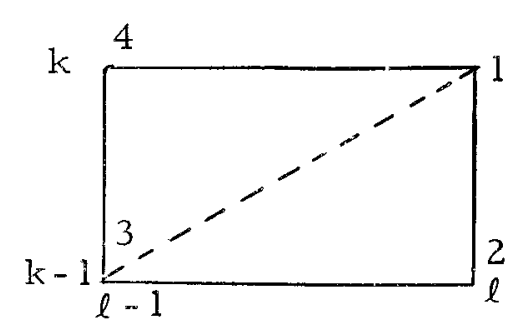

* These expressions are trivially modified in the cartesian case. 
and similar forms for the 134 triangle. (X, $Y=R, Z, u$, or v.) Then

$$
V=\frac{1}{6}\left\{\left(R_{123}\right)\left|\begin{array}{c}
R \\
Z \\
123
\end{array}\right|+\left(R_{134}\right)\left|\begin{array}{c}
R \\
Z \\
134
\end{array}\right|\right\}
$$

and the change of volume from cycle $n$ to cycle $n+1$ is

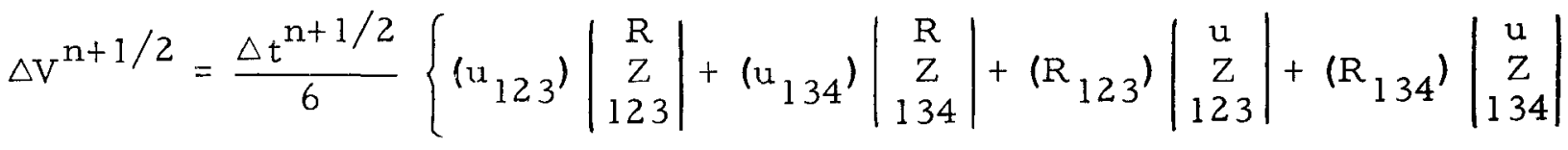

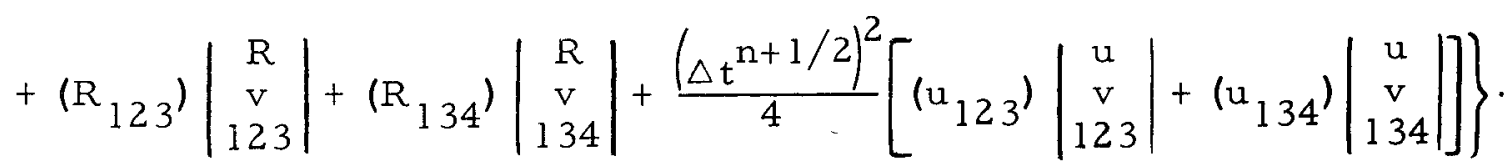

If $\delta V^{n}=V^{n}-V^{0}$ then $\delta V^{n+l}=\delta V^{n}+\Delta V^{n+1 / 2}$

and compression $=(\eta)^{\mathrm{n}+1}=1-\frac{(\delta V)^{\mathrm{n}+1}}{\mathrm{~V}^{\mathrm{n}+1}}$.

In the code, each zone carries a label which indicates what material the zone contains and, consequently, how the stress is to be calculated. The simplest case is that of a hydrodynamic fluid. In this case, all calculations of strain rates are omitted. The deviatoric stress elements $\tau_{i}$ are zero and the pressure is calculated by a simple analytic fit

$$
P=A(\eta)+B(\eta) E .
$$

In all other cases (elastic, plastic, fractured material) the calculation first determines what change in stress would be produced in perfectly elastic material by the deformation which occurred during the previous $\Delta t$ cycle. The resulting stress may then be modified to account for various kinds of inelastic behaviour (plastic flow or fracturej.

The following terms will be needed for calculating strain rates:

$$
\begin{aligned}
2 J^{n+1 / 2} & =\left|\begin{array}{c}
R \\
Z \\
123
\end{array}\right|+\left|\begin{array}{c}
R \\
Z \\
134
\end{array}\right|, \quad \frac{u}{R}=\frac{u_{1}+u_{2}+u_{3}+u_{4}}{R_{1}+R_{2}+R_{3}+R_{4}} \\
\frac{\partial u}{\partial R} & =\frac{1}{2 J}\left\{\left|\begin{array}{c}
u \\
Z \\
123
\end{array}\right|+\left|\begin{array}{c}
u \\
Z \\
134
\end{array}\right|\right\}, \quad \frac{\partial u}{\partial Z}=\frac{1}{2 J}\left[\left(u_{2}-u_{4}\right)\left(R_{1}-R_{3}\right)-\left(u_{1}-u_{3}\right)\left(R_{2}-R_{4}\right)\right],
\end{aligned}
$$




$$
\frac{\partial v}{\partial Z}=\frac{1}{2 J}\left[\left|\begin{array}{c}
R \\
v \\
123
\end{array}\right|+\left|\begin{array}{c}
R \\
v \\
134
\end{array}\right|\right], \frac{\partial v}{\partial R}=\frac{1}{2 J}\left[\left(v_{1}-v_{3}\right)\left(Z_{2}-Z_{4}\right)-\left(v_{2}-v_{4}\right)\left(Z_{1}-Z_{3}\right)\right] .
$$

We define the strain rates:

$$
\begin{aligned}
& \dot{e}_{R R}=\frac{\partial u}{\partial R}, \quad \dot{e}_{Z Z}=\frac{\partial v}{\partial Z}, \quad \dot{e}_{\theta \theta}=\frac{u}{R} \\
& \dot{e}_{R Z}=\frac{1}{2}\left(\frac{\partial u}{\partial Z}+\frac{\partial v}{\partial R}\right), \text { and } \dot{\theta}=\dot{e}_{R R}+\dot{e}_{\theta \theta}+\dot{e}_{Z Z}
\end{aligned}
$$

where $\Theta$ is the dilatation rate. If the strain rates are expressed in deviatoric form:

$$
\begin{aligned}
& \dot{\mathrm{e}}_{R}=\dot{\mathrm{e}}_{R R}-\frac{\dot{\Theta}}{3}=\frac{1}{3}\left(2 \frac{\partial u}{\partial R}-\frac{\partial v}{\partial Z}-\frac{u}{R}\right) \\
& \dot{e}_{Z}=\dot{e}_{Z Z}-\frac{1}{3} \dot{\Theta}=\frac{1}{3}\left(2 \frac{\partial v}{\partial R}-\frac{\partial u}{\partial R}-\frac{u}{R}\right) \\
& \dot{e}_{\theta}=\dot{e}_{\theta \theta}-\frac{1}{3} \dot{\Theta}=-\left(\dot{e}_{R}+\dot{e}_{Z}\right)
\end{aligned}
$$

then Hooke's law becomes:

$$
\begin{aligned}
\dot{\mathrm{P}} & =-\mathrm{k} \dot{\Theta} \\
\dot{\tau}_{i} & =2 \mu \dot{\mathrm{e}}_{\mathrm{i}}(i=R, Z, R Z)
\end{aligned}
$$

where $k$ and $\mu$ are the bulk and rigidity moduli.

In the machine calculation, these equations for $P$ and $\tau_{i}$ are slightly modified. The relationship between pressure and compression is generalized to $P=P(\eta-1)$ which may be a linear function corresponding to constant bulk modulus or, if necessary, may represent a variable compressibility.

The expression for $\tau_{i}$ is modified to include a small correction term for body rotation. If, during the past cycle, the zone has rotated through a small angle

$$
\Delta \phi=\Delta t \text { curl } \vec{u}=\Delta t\left(\frac{\partial u}{\partial Z}-\frac{\partial v}{\partial R}\right),
$$

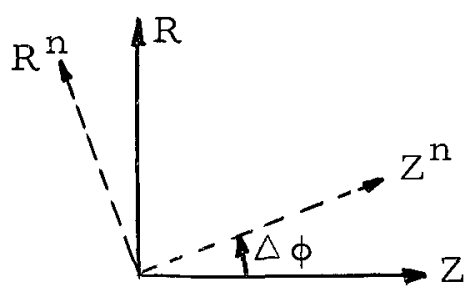


then the stress has rotated with the material. To obtain the stress components in the fixed RZ coordinate system, one must find the RZ components of this slightly rotated stress tensor. Performing the rotation (through $-\Delta \phi$ ) we find

$$
\begin{aligned}
& \tau_{R R}=\tau_{R R}^{n} \cos ^{2} \Delta \phi+\tau_{Z Z}^{n} \sin ^{2} \Delta \phi+2 \tau_{R Z}^{n} \sin \Delta \phi \cos \Delta \phi \\
& \tau_{Z Z}=\tau_{R R}^{n} \sin ^{2} \Delta \phi+\tau_{Z Z}^{n} \cos ^{2} \Delta \phi-2 \tau_{R Z}^{n} \sin \Delta \phi \cos \Delta \phi \\
& \tau_{R Z}=\left(\tau_{Z Z}^{n}-\tau_{R R}^{n}\right) \sin \Delta \phi \cos \Delta \phi+\tau_{R Z}^{\mathrm{n}}\left(\cos ^{2} \Delta \phi-\sin ^{2} \Delta \phi\right) . \\
& \tau_{\theta \theta}=\tau_{\theta \theta}^{n} .
\end{aligned}
$$

This causes the following changes in the deviatoric stress elements (here $\Delta^{\mathrm{ROT}} \tau=$ correction $=\left(\tau^{\text {corrected }}-\tau^{\mathrm{n}}\right)$

$$
\begin{aligned}
\Delta^{\mathrm{ROT}} \tau_{\mathrm{R}} & =\left(\tau_{Z}^{\mathrm{n}}-\tau_{\mathrm{R}}^{\mathrm{n}}\right) \sin ^{2} \Delta \phi+2 \tau_{\mathrm{RZ}}^{\mathrm{n}} \sin \Delta \phi \cos \Delta \phi \\
\Delta^{\mathrm{ROT}} \tau_{Z} & =-\Delta^{\mathrm{ROT}} \tau_{R} \\
\Delta^{\mathrm{ROT}} \tau_{R Z} & =\left(\tau_{Z}^{\mathrm{n}}-\tau_{R}^{\mathrm{n}}\right) \sin \Delta \phi \cos \Delta \phi-Z \tau_{R Z}^{\mathrm{n}} \sin ^{2} \Delta \phi .
\end{aligned}
$$

For small $\Delta t$ it is as sumed that this rotation is independent of the distortion which caused strain changes during $\Delta t$. Thus the net change in stress is taken to be just the sum of distortion plus rotation changes:

$$
\tau_{i}^{n+1}=\tau_{i}^{n}+2 \mu(\Delta t)^{n+l / 2} \dot{e}_{i}^{n+l / 2}+\Delta^{R O T} \tau_{i} \quad i=R, z, R Z .
$$

This, together with the calculated pressure, defines the stress at $t^{n+1}$ if the deformation was perfectly elastic. If, however, the shear or tensile strength of the material has been exceeded, this stress must modified accordingly. The tests and stress modifications which are used in the code to describe such inelastic behaviour are discussed in the next two sections. 


\section{PLASTIC YIELD}

Plastic yielding arises from the inability of real materials to support arbitrarily large shear stress. In the code such inelastic behaviour may be taken into account by testing and suitably modifying the stress during each time cycle. While several proposed yield criteria may be found in the literature, the differences between them are usually much smaller than the uncertainties of the applicable material constants. A simple and convenient choice is a generalized form of the von Mises yield criterion,

$$
f=J_{2}-K^{2}
$$

Here $J_{2}$ is an invariant of the deviatoric stress

$$
\mathrm{J}_{2}=\tau_{\mathrm{R}}^{2}+\tau_{\mathrm{R}}{ }^{\tau} \mathrm{Z}+\tau_{\mathrm{Z}}^{2}+\tau_{\mathrm{RZ}}^{2}
$$

and $\mathrm{K}$ corresponds to the shear strength which may be made a function of pressure and also of work done in previous plastic deformaticn (work hardening). If $f<0$, the material behaves elasticaljy, but if $f>0$, the shear strength has been exceeded and the stress must be adjusted to bring $f$ to zero.

$$
f=0 \text { corresponds to a surface }
$$
defining the yield limit in stress space. The adjustment used (flow rule) moves the stress state back to the surface along the normal, i。e., if during the past cycle the stress changed from

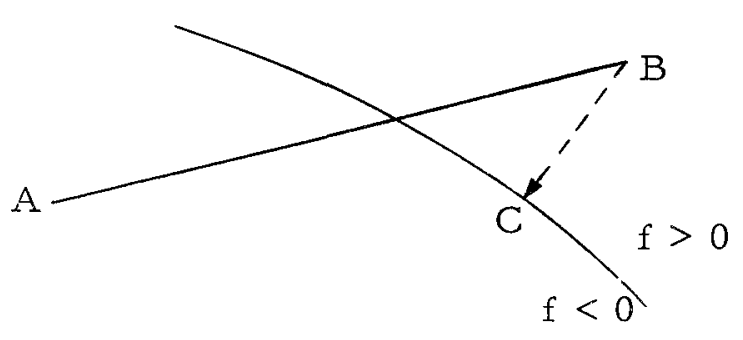
$\tau^{\mathrm{n}}$ (represented by point $\mathrm{A}$ in the sketch) to the (unadjusted) stress $\tau^{n+1}$ at point $B$ then the adjusted stress at $\tau^{n+1}$ is at $C$. In terms of the unadjusted $\tau$ and $J_{2}$ this gives

$$
\tau_{i}^{\text {adjusted }}=\sqrt{\frac{\mathrm{K}^{2}}{\mathrm{~J}_{2}}} \tau_{\mathrm{i}} \quad \mathrm{i}=\mathrm{R}, \mathrm{Z}, \mathrm{RZ} \text {. }
$$

The "plastic strain" is the difference between the total strain which produced the excessive stress at point $B$ and the strain which would just take the stress to the final adjusted value of $\mathrm{C}$. The work done in this plastic deformation is the limiting stress times the plastic strain. This is incremented from cycle to cycle by 


$$
\mathrm{E}_{\text {plast }}^{\mathrm{n}+1}=\mathrm{E}_{\text {plast }}^{\mathrm{n}}+\frac{\mathrm{J}_{2}}{\mu}\left[\sqrt{\frac{\mathrm{K}^{2}}{\mathrm{~J}_{2}}}-\frac{\mathrm{K}^{2}}{\mathrm{~J}_{2}}\right] .
$$

Since shear failure may alter the properties of the material, the code has provisions for re-labeling the material in a zone when it yields for the first time. Thus, one may construct a crude model of a brittle but initially strong material by giving it fairly large constant $K$ but requiring that it be relabeled when first yield occurs. The resulting new material might then have the properties of shattered rock (for example a shear strength simply proportional to pressure and no tensile strength).

Obvious generalizations of the yield conditions are available. K may be made a function of the pressure (a generalized von Mises-Coulomb yield condition), of $E_{\text {plast }}$ (one form of work hardening), of the internal energy $E$ (heat effects), or of the strain rate. The yield conditions may apply to the "elastic" stresses or viscous stresses or both. A Tresca condition ${ }^{\top}$ shear $<\mathrm{K}$ can be treated by the same kind of multiplication of the deviatoric stresses to make the adjusted stress system lie on a yield surface. It should be noted that the treatment of yield allows free motion of the stress state tangent to the yield surface, i. e., the principal axes of stress and strain can change freely with time.

\section{FRACTURE}

When the tensile stress across some element of material exceeds the material's tensile strength, a crack forms normal to the direction of this stress. This direction is not, in general, parallel to either of the $R Z$ coordinate axes used in the code. One can show that for any local stress system there exists a coordinate system such that the stress tensor, when transformed to this system, is diagonal. The elements of this transformed tensor correspond to the greatest (and least) normal stress experienced by the material. It is this greatest stress which will cause the first crack to form and the orientation of the transformed coordinate system corresponds to the direction of the crack. In the code the behaviour of cracked material is simulated by adjusting the stress in the zone so that there is no normal stress across the crack. Thus, while a Lagrange zone representing fractured material does not actually separate into several pieces, the stress in the 
zone is adjusted to the value appropriate to such cracked material. The code also calculates the strain change associated with this adjustment. This strain change is proportional to the width of the crack. In this way one can keep track of the crack width and, until the crack closes again at some later time, continue to adjust the stresses to maintain the component normal to the crack at zero.

The transformation of the stress tensor from the code's $\theta$ RZ system to a rotated system of coordinates 1,2 , 3 (axis 1 parallel to $\theta$ axis) is given by $T_{11}=-\left(P+\tau_{R}+\tau_{Z}\right)$

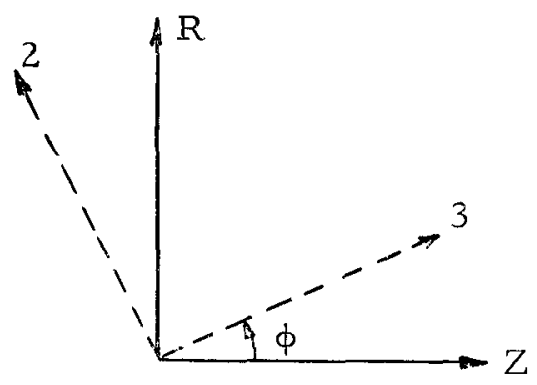
$\mathrm{T}_{22}=-\mathrm{P}+\tau_{\mathrm{R}} \cos ^{2} \phi+\tau_{Z} \sin ^{2} \phi-2 \tau_{R Z} \sin \phi \cos \phi$ $T_{33}=-P+\tau_{R} \sin ^{2} \phi+\tau_{Z} \cos ^{2} \phi+2 \tau R Z \sin \phi \cos \phi$ $T_{23}=\left(\tau_{R}-\tau_{Z}\right) \sin \phi \cos \phi+\tau_{R Z}\left(\cos ^{2} \phi-\sin ^{2} \phi\right)$ where $\phi$ is the angle between tie 3 and $Z$ axes and $T_{i j}$ are the elements of the complete (not deviatoric) stress tensor. When $\phi$ is chosen to give $\mathrm{T}_{23}$ $=0$ we obtain

$$
\begin{aligned}
& T_{11}=-\left(P+\tau_{R}+\tau_{Z}\right) \\
& T_{22}=T_{33}=-P+\frac{1}{2}\left[\tau_{R}+\tau_{Z} \pm \sqrt{\left(\tau_{R}-\tau_{Z}\right)^{2}+\left(2 \tau_{R Z}\right)^{2}}\right] \\
& T_{23}=0, \quad \phi=\frac{1}{2} \tan ^{-1}\left(\frac{2 \tau_{R Z}}{\tau_{Z}-\tau_{R}}\right) .
\end{aligned}
$$

These ("principal") stresses are the greatest and least stresses experienced by the material and this particular set of coordinates is called the principal coordinate system. In the code's treatment of fracture, the principal stresses (in unbroken material) are compared with the material's tensile strength. If one of them exceeds the tensile strength, one must adjust the values of $P$, ${ }^{\tau} R, \tau_{Z}$ and $\tau_{R Z}$ in such a way that the adjusted values represent the proper stress in the fractured material. The choice of this adjustment is based on the following set of assumptions: 
a. The principal stresses can be related to a set of principal strains $E_{i i}$ by Lamé constants

$$
T_{i i}=\lambda \Theta+2 \mu E_{i i} \text { where } \Theta=E_{11}+E_{22}+E_{33} .
$$

Thus, the stress-strain relation is assumed to be linear in the range encountered by fractured material.

b. We ignore the possibility of gas-filled fractures and, therefore, require that the stress normal to the crack surface be zero.

c. When a fracture suddenly opens (or an old crack grows or shrinks), the resulting motion of material is assumed to be normal to the crack surface. If, for example, the principal stress $T_{33}$ exceeded the tensile strength, the principal strain $E_{33}$ will be adjusted to $\mathrm{E}_{33}+\Delta \mathrm{E}_{33}$ and the other principal strains will not be altered. The adjusted principal stresses will then be:

$$
\begin{aligned}
& T_{11}^{\prime}=T_{11}+\lambda \Delta E_{33} \\
& T_{22}^{\prime}=T_{22}+\lambda \Delta E_{33} \\
& T_{33}^{\prime}=0 \\
& \text { where } \Delta E_{33}=\frac{-T_{33}}{\lambda+2 \mu} .
\end{aligned}
$$

d. It is assumed that a crack rotates with the material (amount of body rotation per cycle $=\Delta \phi$ ) and that the creation of a crack or its growth does not rotate the principal coordinates. One may now transform the adjusted stress tensor $\left(T_{1}, T_{2}, T_{3}\right)$ back to the $R Z$ coordinate system by rotation through $-\phi$. Since $\mathrm{T}_{23}^{\prime}$ is zero:

$$
\begin{aligned}
P^{\prime} & =-\frac{1}{3}\left(T_{1}^{\prime}+T_{2}^{\prime}+T_{3}^{\prime}\right) \\
\tau_{R}^{\prime} & =P^{\prime}+T_{2}^{\prime} \cos ^{2} \phi+T_{3}^{\prime} \sin ^{2} \phi \\
\tau_{Z}^{\prime} & =P^{\prime}+T_{2}^{\prime} \sin ^{2} \phi+T_{3}^{\prime} \cos ^{2} \phi \\
\tau_{R Z}^{\prime} & =\left(T_{3}^{\prime}-T_{2}^{\prime}\right) \sin \phi \cos \phi .
\end{aligned}
$$


The amount of adjustment $\left(\Delta E_{33}\right)$ is a measure of the change of crack width during this cycle. The code carries a set of variables $\left(E_{1}, E_{2}, E_{3}\right)$ which correspond to the sum of past adjustments. Thus, on each subsequent cycle it can recognize the presence of an old type-3 crack by $E_{3} \neq 0$. If it finds that $E_{3}<0$ then $T_{33}$ must be adjusted to zero and the new value of $E_{3}$ is $E_{3}$ old $+\triangle E_{33}$. If, however, the new $E_{3}$ is positive, the crack has closed, and the adjustment is made with $\Delta E_{33}=-E_{3}^{\circ}$ (this will not bring $\mathrm{T}_{33}$ completely to zero).

It is, of course, possible that two mutually perpendicular cracks exist simultaneously in the same element of material. Therefore, following the adjustments described above, the remaining adjusted principal stresses must be compared with the tensile strength. If a second crack has formed the process of stress adjustment is essentially the same as before. If, for example, both $E_{1}$ and $E_{2}$ are negative, one must adjust $T_{11}$ and $T_{22}$ to zero by

$$
\begin{aligned}
& T_{11}^{\prime}=0, \quad T_{22}^{\prime}=0, \\
& T_{33}^{\prime}=T_{33}+\lambda\left(\Delta E_{11}+\Delta E_{22}\right)
\end{aligned}
$$

where

$$
\Delta E_{11}=\frac{\lambda T_{22}-(\lambda+2 \mu) T_{11}}{2 \mu(2 \lambda+2 \mu)}, \Delta E_{22}=\frac{\lambda T_{11}-(\lambda+2 \mu) T_{22}}{2 \mu(2 \lambda+2 \mu)}
$$

and then transform back to $R Z$ coordinates as before. After this adjustment, the remaining nonzero principal stress is tested for fracture. If a third crack has formed, all the element of the stress tensor must vanish (physically this corresponds to something like uncompressed gravel). When this occurs, all stress terms $\left(P, \tau_{i}\right)$ and crack widths $\left(E_{i}\right)$ are set to zero and the material label of the zone is changed. On subsequent cycles, the material is treated like "sand" (zero tensile strength, shear strength proportional to pressure).

One potential error in this model of fracture arises from the code's inability to take account of anisotropy caused by the presence of formerly open cracks. When a crack closes, the tensile strength is assumed to be unchanged (i.e., a closed crack is assumed to have "healed"). In underground explosion calculations, this problem is not serious, because rocks, 
on the very large scale involved, have almost no tensile strength (i.e., we assume that there are many, random, pre-existing closed cracks). It is possible, however, to re-label a zone when it first cracks and subsequently treat it as a zero-strength material, as in the treatment of brittle shear failure described in the previous section.

A point concerning the normal pressure calculation should be mentioned. When pressure is calculated as a function of compression, it is the compression of the material that matters. This is the compression of the Lagrangian zone corrected for the size of any open fractures that may exist in the zone. Therefore, the pressure is calculated as

$$
P=P(\eta-1)_{\text {effective }}
$$

where

$$
(\eta-1)_{\text {effective }}=(\eta-1)_{\text {zone }}-E_{1}-E_{2}-E_{3} .
$$

\section{VIII.DAMPING}

An artificial viscosity is used to damp out spurious oscillations. It is analogous to the artificial viscosity first introduced by Von Neumann and Richtmyer to permit numerical calculation of shock hydrodynamics by replacing the discontinuous pressure jump across a shock by a rapid but continuous change. In hydrodynamics, the damping term has the form

$$
q=\operatorname{Ap}(\Delta u)^{2}
$$

where $\Delta \mathrm{u}$ is the velocity difference across the zone and $\mathrm{A}$ is a dimensionless constant $\simeq 1 / 2$. This form, while satisfactory for strong shocks in $\gamma$-law fluids, provides insufficient damping for sonic signals in elastic solids (unless the constant $A$ is assigned a very large value). Effective damping in the sonic range is obtained by using terms linear in velocity differences instead of the quadratic form shown above.

The quadratic $q$ spreads the pressure and velocity discontinuity at a shock front over a limited number of zones (typically three), and this number remains constant as the wave is transmitted through the mesh. The use of linear damping terms unfortunately causes an initially sharp signal to spread without limit. This effect would probably cause difficulties in calculations 
involving the passage of a very sharp wave front through a very large number of zones. In most past calculations, however, this has not presented a serious problem. The signal diffused quite slowly and the stress wave retained its shape reasonably well.

Like the stress tensor, the damping terms are expressed as the sum of a scalar term, $Q$, and a deviatoric tensor with elements $Q_{R}, Q_{Z}, Q_{R Z}$. Several forms for $Q$ are availabie in the calculation, although they would not all be used simultaneously. The scalar term is calculated as

$$
Q=Q_{1}+Q_{2}+Q_{3}
$$

where

$$
\begin{aligned}
& Q_{1}=C_{1} \rho(\Delta U)^{2} \\
& Q_{2}=C_{2} C \rho \sqrt{\Delta U^{2}} \\
& Q_{3}=C_{3} C \rho \Delta X \frac{1}{V} \frac{\partial V}{\partial t} .
\end{aligned}
$$

Here $Q_{1}$ is the usual (quadratic) Von Neumann $Q_{8}$

$Q_{2}$ is a linear form of $Q_{1}$ which is only effective at low velocities,

$Q_{3}$ is a volume viscosity which together with the deviatoric terms $Q_{i}$ corresponds to the stress tensor in viscous flow.

$C_{1}, C_{2}, C_{3}$ are dimensionless constants $(\sim 1 / 2)$,

$C$ and $\rho$ are sound speed and density,

$\Delta \mathrm{X}$ is the shortest distance across the zone,

$\mathrm{V}$ is the zone volume

and $\Delta U$, the average velocity difference across the zone, is calculated only when the zone is collapsing but is set to zero when the zone is expanding.

The deviatoric tensor terms are

$$
Q_{i}=C_{4} C \rho \Delta X \dot{e}_{i}(i=R, Z, R Z)
$$

where the $\stackrel{\circ}{i}_{i}$ are the three deviatoric strain rates described previously. 
The artificial viscosity tensor effectively damps only those velocity oscillations which would produce variations in the stress. In some calculations, however, a velocity pattern like the one shown in the sketch was encountered. (The velocities shown are the variations from the smooth velocity

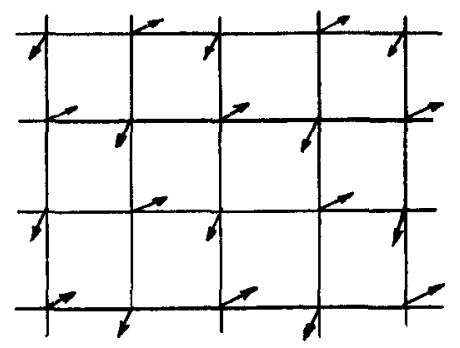
field that was expected.) This pattern was first noticed in a calculation where a spherical elastic wave was propagated through a rectangular mesh. The same pattern, but with smaller amplitude, was found when the material was treated as a fluid. This velocity noise characteristically has equal velocities at opposite corners of the zones $\left(u_{1}=u_{3}\right.$ and $\left.u_{2}=u_{4}\right)$ and is not damped by the strain-rate dependent $Q$ described above* ${ }^{*}$ It is, however, readily damped by a $Q$ term dependent on the relative rate of rotation of opposite sides of a zone.

The rate of rotation of a zone side $(\mathrm{e} . \mathrm{g} ., 1,4)$ is given by

$\omega_{14}=\frac{\left(u_{1}-u_{4}\right)\left(z_{1}-z_{4}\right)-\left(v_{1}-v_{4}\right)\left(R_{1}-R_{4}\right)}{\left(R_{1}-R_{4}\right)^{2}+\left(z_{1}-z_{4}\right)^{2}}$

Let

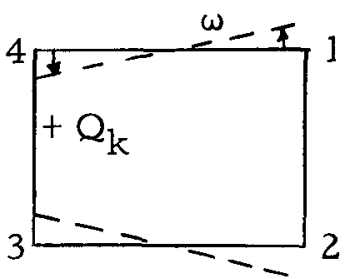

$Q_{k}=C_{5} C \rho \Delta X \frac{\partial \omega}{\partial k}=\left(C_{5} C \rho \Delta X\right)\left(\omega_{14}-\omega_{23}\right)$

$Q_{\ell}=-\left(C_{5} C \rho \Delta X\right) \frac{\partial \omega}{\partial \ell}=\left(C_{5} C \rho \Delta X\right)\left(\omega_{43}-\omega_{12}\right)$

the signs being chosen so that $Q$ acts as

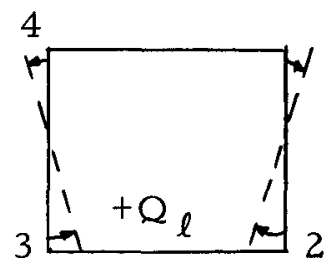

a positive pressure when viewed from

point 3 and as a tension when viewed

from the opposite corner of the zone. The resulting accelerations (described as "q terms" on pages 9 and 10).

$$
\text { (i) }{ }_{61} q=\frac{1}{(\rho J)_{61}} \quad\left[\left(Q_{\ell}\right)_{C-B} \bar{Z}_{6 l}\right]
$$

* The strain rates calculated in each zone may be defined entirely in terms of the rates of change of the dot and cross products of the vectors connecting diagonally opposite corners of a zone. The velocity noise under discussion corresponds to the relative translation of these diagonal vectors and, therefore, produces no strain rate. 


$$
\begin{array}{ll}
(\dot{v})_{61}{ }^{q}=\frac{-1}{(\rho J)_{61}} & {\left[\left(Q_{\ell}\right)_{C-B} \bar{R}_{61}\right]} \\
(\dot{u})_{81}{ }^{q}=\frac{-1}{(\rho J)_{81}} & {\left[\left(Q_{k}\right)_{C-D} \bar{Z}_{81}\right]} \\
(\dot{v})_{81}{ }^{q}=\frac{1}{(\rho J)_{81}} \quad\left[\left(Q_{k}\right)_{C-D} \bar{R}_{81}\right]
\end{array}
$$

when viewed from the other end of the zone edge, the signs are reversed so that

$$
\text { (i) }{ }_{12}^{\mathrm{q}}=\frac{-1}{(\rho \mathrm{J})_{12}} \quad\left[\left(\mathrm{Q}_{\ell}\right)_{\mathrm{D}-\mathrm{A}} \overline{\mathrm{Z}}_{12}\right] \text {, etc. }
$$

IX. ENERGY EQUATION AND STABILIT Y CONTROL

The change in the internal energy of each zone is calculated during each cycle. For elastic-plastic materials, where the stress is not a function of internal energy, this calculation is not really necessary. The conservation of the total energy in the entire problem does, however, furnish a valuable indication that the code is operating properly.

In elastic-plastic material, the internal energy (per unit original volume) is incremented from cycle to cycle by $E^{n+1}=E^{n}+\left(2 \bar{\tau}_{R}+\bar{\tau}_{Z}\right) \Delta e_{R}+\left(\bar{\tau}_{R}+2 \bar{\tau}_{Z}\right) \Delta e_{Z}+2 \bar{\tau}_{R Z} \Delta e_{R Z}-\bar{P} \frac{\Delta V}{V^{0}}$

where the time-centered stress terms include the damping tensor:

$$
\begin{aligned}
\bar{P} & =1 / 2\left(P^{n}+P^{n+1}\right)+Q^{n+1 / 2}, \\
\bar{\tau}_{R} & =1 / 2\left(\tau_{R}^{n}+\tau_{R}^{n+1}\right)+Q_{R}^{n+1 / 2}, \text { etc. }
\end{aligned}
$$

The $\Delta e_{R}, \Delta e_{Z}, \Delta e_{R Z}$ are the change in strain during the past cycle, and $\Delta V$ is the change of zone volume. The work done by the $Q_{K}$ and $Q_{L}$ terms (described in the previous section) has been ignored.

In fluid materials, the deviatoric tensor terms are zero and the pressure is calculated by a simple polynomial form

$$
P=A(\eta)+B(\eta) E
$$


where

$$
\begin{aligned}
& \mathrm{A}(\eta)=\mathrm{a}_{0}+\mathrm{a}_{1}(\eta-1)+\mathrm{a}_{2}(\eta-1)^{2}+\mathrm{a}_{3}(\eta-1)^{3} \\
& \mathrm{~B}(\eta)=\mathrm{b}_{0}+\mathrm{b}_{1}(\eta-1)+\mathrm{b}_{2}(\eta-1)^{2}
\end{aligned}
$$

and the zone compression

$$
\eta^{n+1}=1-\frac{\delta V^{n+1}}{V^{n+1}}
$$

The internal energy $E^{n+1}=E^{n}-\bar{P} \Delta V^{n+l} / 2 / V^{0}$ is expressed in terms of $\mathrm{P}^{\mathrm{n}}$ and $\eta^{\mathrm{n}+1}$ as

$$
E^{n+1}=\frac{1}{1+\frac{B(\eta)}{2} \frac{\Delta V}{V^{0}}}\left[E^{n}-\left(Q+\frac{P^{n}+A(\eta)}{2}\right) \frac{\Delta V^{n+1 / 2}}{V^{0}}\right]
$$

To ensure stability in the finite difference equations, the time step $\Delta t$ must be limited. In a fluid, with no damping terms, one can show that a perturbation will neither grow nor shrink if $\Delta t \leq \Delta \mathrm{L} / \mathrm{C}$ where $\Delta \mathrm{L}$ is the zone width and $C$ is the sound speed (i.e., $\Delta t$ must be less than the time required for a sound signal to cross the zone). The presence of damping terms results in a slightly more stringent stability condition. We have not analyzed the stability condition in the present case of tensor forces, and such complications as fracture and plastic flow. Satisfactory stability has been obtained by limiting $\Delta t$ to about half of the above value.

In an elastic-plastic material, the dilatational sound speed is used:

$$
C^{2}=\frac{1}{\rho}(\lambda+2 \mu)
$$

In a zone containing a hydrodynamic fluid:

$$
\mathrm{C}^{2}=\frac{1}{\rho^{0}}\left[\frac{\partial \mathrm{A}(\eta-1)}{\partial(\eta-1)}+\mathrm{E} \frac{\partial \mathrm{B}(\eta-1)}{\partial(\eta-1)}+\frac{\mathrm{PB}(\eta-1)}{\eta^{2}} .\right.
$$

For each zone a maximum permissible time step

$$
\Delta \mathrm{t}=\mathrm{K}_{0} \frac{\Delta \mathrm{L}}{\mathrm{C}} \quad\left(\mathrm{K}_{0} \approx 1 / 2\right)
$$

is calculated and the smallest of these is then used as $\Delta t$ for the entire mesh during the following cycle. 


\section{EXAMPLES}

In this section two examples of calculations are presented to illustrate the uses and limitations of the code. They are one-dimensional and served as test problems by permitting comparison with analytic solutions.

\section{A. Spherical Elastic Wave}

As the first example, we consider the problem of the stress wave generated in an infinite elastic medium by an exponentially decaying pressure in a spherical cavity. The analytic solution for this problem has been given by Blake. ${ }^{6}$ Several TENSOR calculations were done with various zone sizes, damping constants, etc., and were then compared with the Blake solution.

The zone mesh for one of these problems is shown in Fig. 1. The spherical geometry has been approximated by a polygon of revolution. The "angular width" of the zones $(3$ degrees in this case) has almost no effect on the resulting motion. A comparison problem using 10-degree zones gave velocities, stress, etc., which differed by less than one percent from those in the 3-degree problem.

Rigid wall boundary conditions were used at $\ell=0$ and $\ell_{\max }=3$ and a pressure history of the form

$$
\left.P=e^{-t} \text { kilobars ( } t \text { in } \mathrm{msec}\right)
$$

was applied at the inner, $K=0$, surface. The outer boundary $\left(K_{\max }=40\right)$ was a free surface. This, of course, limited the time during which the problem could follow the infinite medium solution. The elastic constants are listed in Fig. 1. Damping constants $C_{3}=0.1333$ and $C_{4}=0.1$ were used. (This is equivalent to a damping value of 0.2 for dilatational waves.) The effect of zone size is shown in a second problem, A-l, using the same constants but twice the number of radial zones $\left(K_{\max }=80\right)$.

The resulting velocities and displacements are shown in Figs. 2 and 3, together with the values expected from the Blake solution. Agreement with the analytic solution is generally quite satisfactory. The only real discrepancy is seen in the initial overshoot and oscillation of the inner surface $(K=0)$ in Fig. 2 and in the diffuse wave front at 20 meters in Fig. 3. The initial

\footnotetext{
${ }^{6}$ F. G. Blake, Jr., J. Acoust. Soc. Am. 24, 211 (1952).
} 


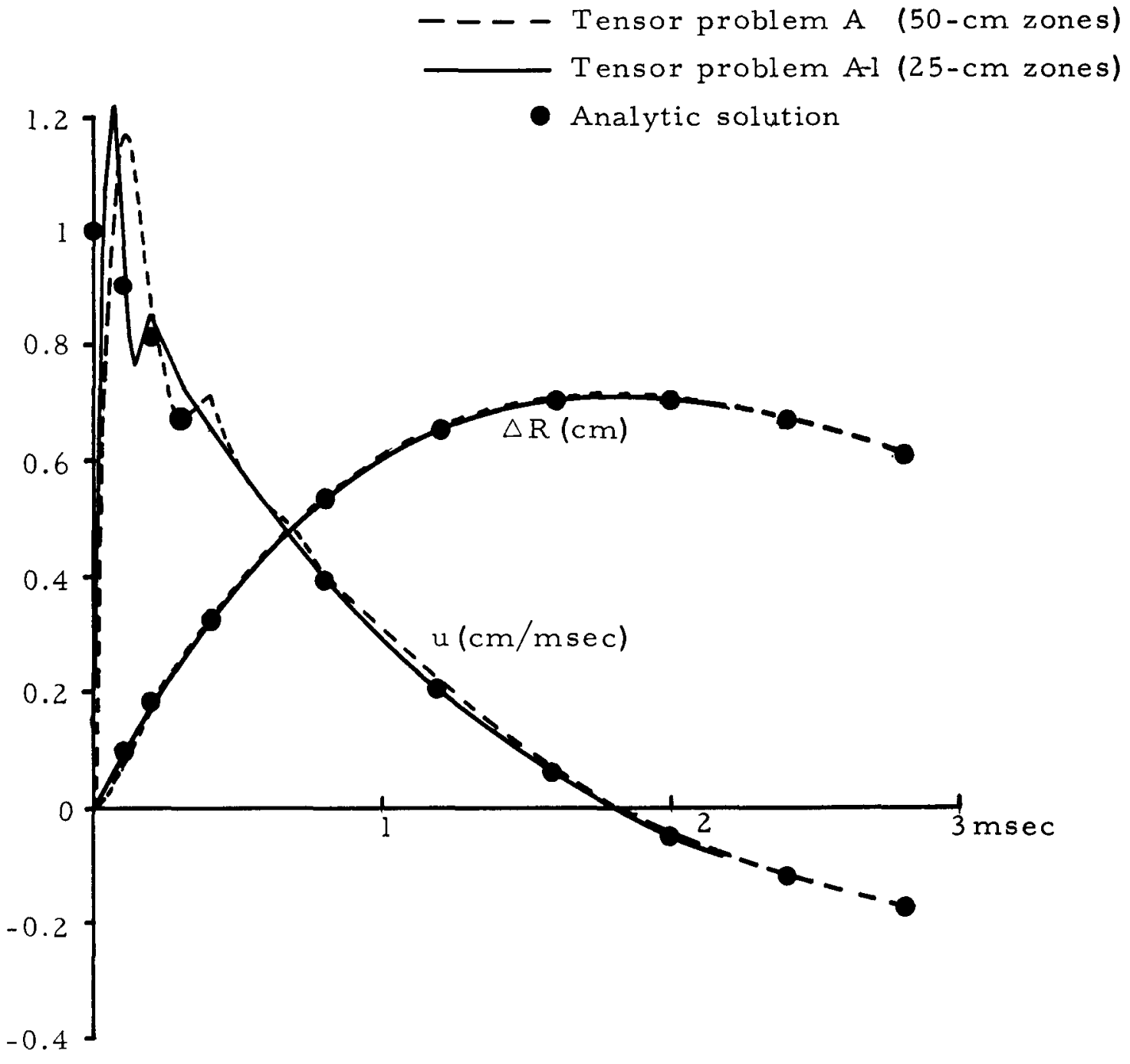

GLL-635-1175

Fig. 2. Inner surface velocity $u$ and displacement $\Delta R$. 

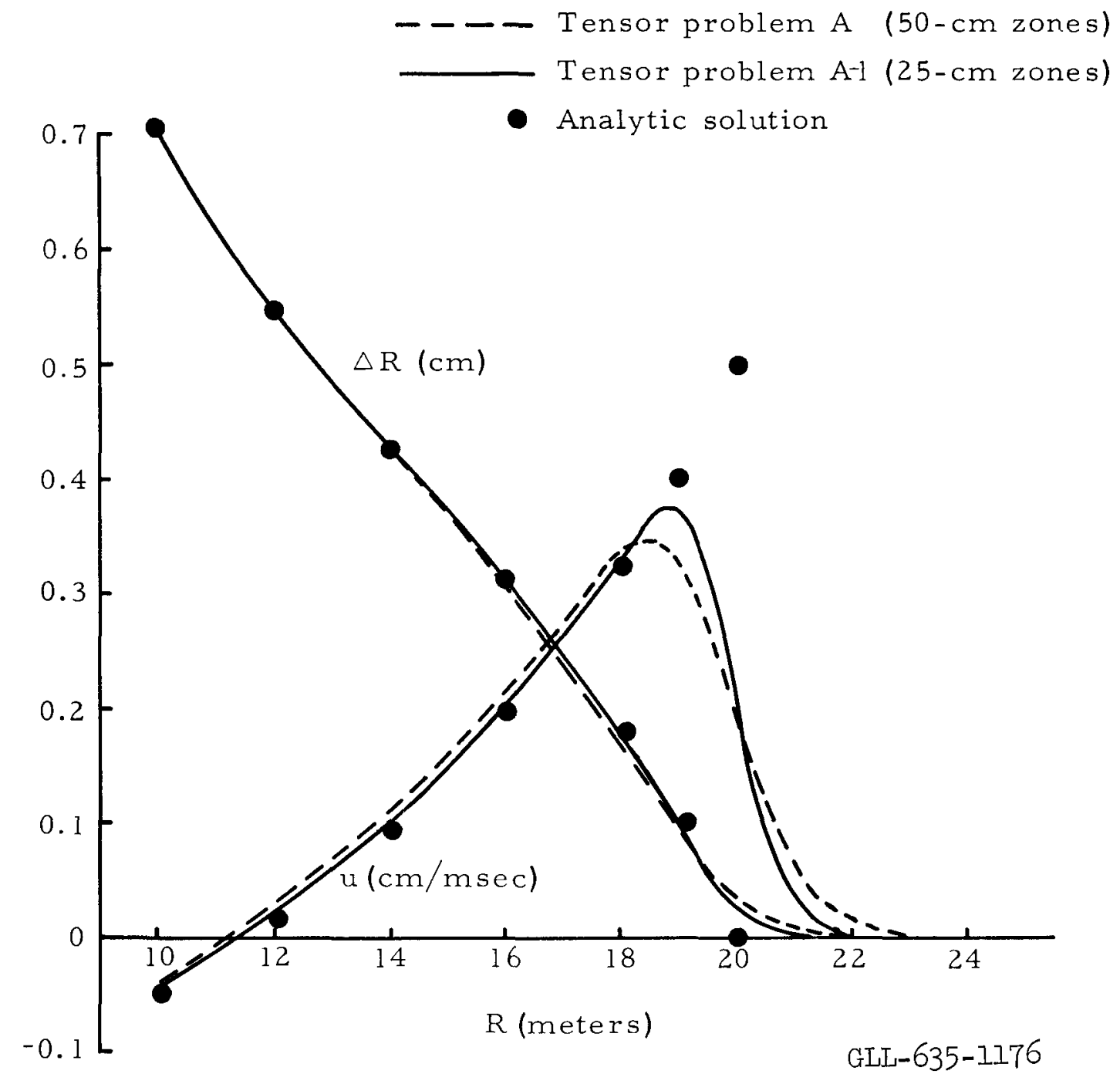

Fig. 3. Radial distributions of velocity and displacement at $t=2 \mathrm{msec}$. 
overshoot can, of course, be damped by use of larger $Q$ constants, but this results in correspondingly greater diffusion of the wave front at later times. Only a mild improvement in the quality of the results is obtained in the finely zoned problem, A-1. This is somewhat reassuring, because in twodimensional calculations, considerations of computer time generally require that rather coarse zoning be used. It shows, however, that while the general motion is calculated quite well, the code will not reproduce velocity and stress peaks which are only a few zones wide. The failure to represent short (in terms of zone size) wave lengths accurately is characteristic of difference schemes of this type and holds even for sonic disturbances in onedimensional fluid flow problems.

\section{B. Plastic Spherical Shell}

To compare the code's treatment of plasticity with an analytic solution, it was necessary to consider a system in static equilibrium. While the code is intended to study transient dynamic effects, no exact analytic solution involving dynamic plastic deformation appeared to be available. Therefore, the test calculation was done on the much simpler static case of a thick spherical shell loaded by an internal pressure sufficient to stress the inner parts to the yield point. ${ }^{7}$ The geometry is shown in the sketch and zoning similar to problem A was used. With

a shear strength of $0.5 \mathrm{~kb}$, the material

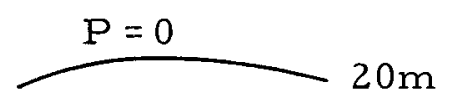
between the inner surface and $14.49 \mathrm{~m}$ should be stressed to the yield point while the outer part of the shell remains elastic.

$$
\widehat{P=1 \mathrm{~kb}} \mathrm{R}=10 \mathrm{~m}
$$

In order to avoid inertial effects, which would cause the code calculation to overshoot the equilibrium position (irreversibly), a "relaxation" method was used. At frequent intervals (one or two msec), all velocities were set to zero. Thus, in a series of brief motions, the shell should gradually approach an equilibrium configuration.

The stress and displacement distributions in the shell at $\mathrm{t}=20 \mathrm{msec}$ are shown in Fig. 4. Since the calculated displacements exceed the

${ }^{7}$ R. Hill, op。cit。 


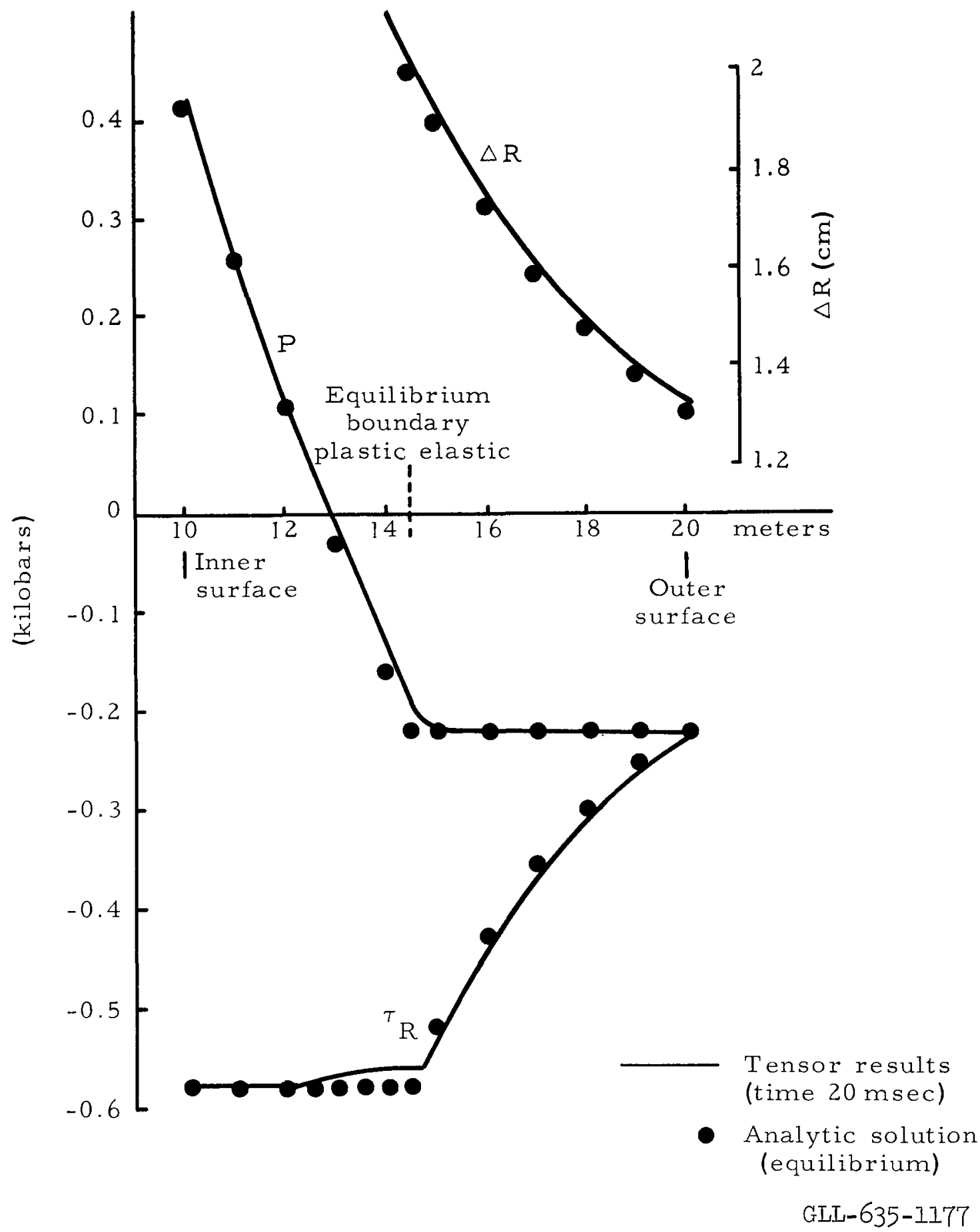

Fig. 4. TENSOR problem B. 
equilibrium values, it is apparent that the velocities should have been set to zero more frequently. The calculated stresses are, however, quite close to the expected equilibrium values and would, presumably, converge on them if the relaxation process were carried further. It was not carried further because the rate of approach to equilibrium becomes extremely slow (the stresses changed by only a few percent during the last $5 \mathrm{msec}$ ). 
This report was prepared as an account of Government sponsored work. Neither the United States, nor the Commission, nor any person acting on behalf of the Commission:

A. Makes any warranty or representation, expressed or implied, with respect to the accuracy, completeness, or usefulness of the information contained in this report, or that the use of any information, apparatus, method, or process disclosed in this report may not infringe privately owned rights; or

B. Assumes any liabilities with respect to the use of, or for damages resulting from the use of any information, apparatus, method, or process disclosed in this report.

As used in the above, "person acting on behalf of the Commission" includes any employee or contractor of the Commission, or employee of such contractor, to the extent that such employee or contractor of the Commission, or employee of such contractor prepares, disseminates, or provides access to, any information pursuant to his employment or contract with the Commission, or his employment with such contractor. 